Original Article

Journal of Epilepsy Research pISSN 2233-6249 / elSSN 2233-6257

Received June 1, 2012

Accepted June 21, 2013

Corresponding author: Dae-Won Seo Department of Neurology, Samsung

Medical Center, 81 Irwon-ro,

Gangnam-gu, Seoul 135-710, Korea

Tel. +82-2-3410-3595

Fax. +82-2-3410-0052

E-mail; daewon@skku.edu

\title{
Propofol Infusion Syndrome in Refractory Status Epilepticus
}

\author{
Woo Sub Hwang ${ }^{1}$, Hye Min Gwak ${ }^{2}$, Dae-Won Seo ${ }^{1}$ \\ Departments of ${ }^{1}$ Neurology and ${ }^{2}$ Obstetrics and Gynecology, Samsung Medical Center, Sungkyunkwan University \\ School of Medicine, Seoul, Korea
}

Background and Purpose: Propofol is used for treating refractory status epilepticus, which has high rate of mortality. Propofol infusion syndrome is a rare but often fatal syndrome, characterized by lactic acidosis, lipidemia, and cardiac failure, associated with propofol infusion over prolonged periods of time. We investigated the clinical factors that characterize propofol infusion syndrome to know the risk of them in refractory status epilepticus.

Methods: This retrospective observation study was conducted in Samsung medical center from Jan. 2005 to Dec. 2009. Thirty two patients (19 males, 13 females, aged between 16 and 64 years), with refractory status epilepsy were included. Their clinical findings and treatment outcomes were evaluated retrospectively. We divided our patients into established status epilepticus (ESE) and refractory status epilepticus (RSE). And then the patients with RSE was further subdivided into propofol treatment group (RSE-P) and the other anesthetics treatment group (RSE-O). We analyzed the clinical characteristics by comparison of the groups.

Results: There were significant differences of hypotension and lipid change between ESE and RSE $(p<0.05)$. However, there was no significant difference between RSE-P and RSE-O groups. The hospital days were longer in RSE than in ESE $(p=0.012)$ and treatment outcome was also worse in RSE than in ESE ( $p=0.007)$ but there were no significant differences of hospital stays and treatment outcome between RSE-P and RSE-O.

Conclusions: RSE is very critical disease with high mortality, which may show as many clinical changes as propofol infusion syndrome. Therefore propofol infusion syndrome might be considered as one of the clinical manifestations of RSE. (2013;3:21-27)

Key words: Propofol infusion syndrome, Status Epilepticus, Anesthetics

\section{Introduction}

The operational definition of status epilepticus is based on the length of time. Recent many trials were introduced, but $9-30 \%$ of status epilepticus remains to be refractory to treatment. ${ }^{1-3}$ Timedependent pharmacoresistance is beneficial and therapeutic design can be used according to a defined protocol. For the stage of status epilepticus, impending, established, refractory status epilepticus can be used. Impending status is the initiation phase of status epilepticus, a disorder in which the duration of seizures is away from the norm of a single seizure. Established status is continuum of impending status, 30 min of continuous seizures. Refractory status is a term that is defined by the failure of adequate amounts of two intravenous drugs to stop seizures, ${ }^{4}$ and lasting more than 2 hours. Refractory status epilepticus develops in $23-44 \%$ of status epilepticus patients and has a mortality of $16-39 \%{ }^{2}$

In refractory status epilepticus, when phenytoin is failed to stop status epilepticus, barbiturate, benzodiazepines, ketamine, and propofol have been used according to treatment guideline of status epilepticus of ILAE and other recent reports. ${ }^{5-7}$ For continuous intravenous therapy for refractory status epilepticus, physicians chose especially midazolam and propofol, rather than a standard AED and then pentobarbital was chosen later in the therapy. ${ }^{7}$ Propofol is a ultrashort-acting sedative-hypnotic agent and has been considered as a promising drug, but may induce excitation and raise the risk of mortality. ${ }^{5}$ The propofol has medium frequency among the 
three drugs in an acute failure, breakthrough seizure, withdrawal seizure, hypotension and minimum risk of mortality. ${ }^{6}$ And propofol has disadvantages of frequent relapses when tapering, and risk of propofol infusion syndrome. ${ }^{8}$ We should monitor the side effects that are hypotension, bradycardia, rhabdomyolysis, and hypertriglycemia. ${ }^{5,9,10}$

The incidence of propofol infusion syndrome is about 39\%, and mortality rate is up to $6 \%$. Propofol infusion syndrome is characterized as the occurrence of acute bradycardia resistant to treatment and progressing to asystole, lipemia, hepatomegaly due to fat infiltration, severe metabolic acidosis, and muscle involvement with evidence of rhabdomyolysis or myoglobinuria and so on. ${ }^{11-13}$ According with other studies, the diagnosis of propofol infusion syndrome can be based on one or more those features ${ }^{11}$ or the presence of bradycardia and at least 1 sign of lipidemia, enlarged liver, metabolic acidosis, or rhabdomyolysis or myoblobulinuria. 14,15 But those clinical features of propofol infusion syndrome are overlapped with those of refractory status epilepticus. ${ }^{16,17}$ If we don't know of use of propofol, it is hard to distinguish propofol infusion syndrome from the unspecific clinical situations by refractory status epilepticus. Actually there are no studies that the presence of disease entity of propofol infusion syndrome in status epilepticus. The purpose of this study is to know whether the clinical findings of propofol infusion syndrome are due to use of propofol or refractory status epilepticus itself.

\section{Subjects and Methods}

\section{Subjects}

We enrolled all the patients who had admitted at Samsung Medical Center and diagnosed with status epilepticus from Jan. 2005 to Dec. 2009. Status epilepticus was defined as no recovery of mental status or seizures over 30 minutes, and RSE is defined as failure of first line and second line treatments with no time limit. Nonconvulsive status epilepticus was defined as presence of EEG of status epilepticus or improved patient with treatment of status epilepticus. We excluded patients without definite electrographic abnormalities or specific clinical features. And the patients who had not currently status epilepticus and under 16 years old were also excluded.

Thirty two patients were included. Female was 13 (40.6\%) and male was 19 (59.4\%). The median age was 28.5 years old (range, 16-81). Among them, 21 (65.5\%) patients had refractory status epilepticus and 11 (34.4\%) patients had status epilepticus that was
Table 1. Clinical features of the subjects with status epilepticus

\begin{tabular}{|c|c|c|c|c|}
\hline & & & $\mathrm{N}$ & Percent \\
\hline \multirow[t]{2}{*}{ Sex } & Female & & 13 & 40.6 \\
\hline & Male & & 19 & 59.4 \\
\hline \multicolumn{2}{|c|}{ Age [yr, median (range)] } & \multicolumn{3}{|c|}{$28.5(16-81)$} \\
\hline \multirow[t]{4}{*}{ Etiology } & infection & & 16 & 50.0 \\
\hline & vascular & & 6 & 18.8 \\
\hline & neoplasm & & 1 & 3.1 \\
\hline & unknown & & 9 & 28.1 \\
\hline \multirow[t]{4}{*}{ Status epilepticus } & ESE & & 11 & 34.4 \\
\hline & RSE & & 21 & 65.5 \\
\hline & & RSE-P & 13 & 61.9 \\
\hline & & RSE-O & 8 & 38.1 \\
\hline \multicolumn{2}{|c|}{ Hospital stays [d, median (range)] } & \multicolumn{3}{|c|}{$12.5(2-240)$} \\
\hline \multirow[t]{3}{*}{ Outcome } & return to baseline & & 8 & 25.0 \\
\hline & no return to baseline & & 15 & 46.9 \\
\hline & death & & 9 & 28.1 \\
\hline Previous epilepsy & & & 10 & 31.3 \\
\hline
\end{tabular}

"ESS: established status epilepticus, RSE-P/O: refractory status epilepticus, treated with propofol/the other anesthetics.

terminated by the first- and second line treatments. The classification of status epilepticus was convulsive type in 31 patients and nonconvulsive type in 1 patient. Among the 21 patients with refractory status epilepticus, 13 (61.9\%) patients were treated with propofol and $8(38.1 \%)$ patients with the other anesthetics such as midazolam and pentobarbital. The median days of hospitalization was 12.5 days (2-240 days). According to the outcome, 9 (28.1\%) patients were death, $15(46.9 \%)$ patients did not return to baseline state, and 11 (25.0\%) patients returned to baseline state. The history of epilepsy was in $10(31.3 \%)$ patients (Table 1).

\section{Methods}

We retrospectively reviewed the patients' medical records. We collected the data on presence of previous history of epilepsy, clinical feature of status epilepticus (convulsive, non-convulsive), the causes of status epilepticus (infection, vascular, neoplasm, unknown), the numbers of concurrent antiepileptic drugs (AED) and clinical features of propofol infusion syndrome including metabolic acidosis, heart abnormalities, rhabdomyolysis, lipid change, hepatic change, renal change, death.

We evaluated the changes of clinical features on each category, based on the diagnostic criteria of lyer et al. ${ }^{11}$ Metabolic acidosis was defined when one or more features of metabolic acidosis was found on arterial blood gases, heart changes when cardiac arrhythmia or 
asystole was on ECG, rhabdomyolysis when blood level of creatine kinase and myoglobulin were increased, lipid change when lipemia was detected on blood tests, hepatic change when elevated hepatic enzymes or abnormal ultrasonographic findings were found, renal change when urine output showed presence of oliguria, anuria, or blood test revealed increased creatinine or potassium. We categorized the outcomes of treatment into 'return to baseline', 'do not return to baseline' and 'dead'.

We classified the patients with status epilepticus into established status epilepticus (ESE) and refractory status epilepticus (RSE) groups. And then the RSE group was further divided into propofol treatment (RSE-P) group and the other anesthetics treatment (RSE-0) group. The other intravenous anesthetics were midazolam and pentobarbital.

\section{Analysis and statistics}

We estimated the overall rate of the data of each clinical parameter. And then we compared those based first on the refractoriness of status epilepticus and then on use of propofol. So we analyzed the differences of clinical features between ESE and RSE groups. Also among RSE patients, RSE-P group was compared to RSE-0 group. Finally we focused on the comparison of hospital days and treatment outcome between RSE-P and RSE-0 groups. We used SPSS 14.0k for statistical analysis. We used Fisher's exact two-tailed test for comparing sex, hypotension, metabolic acidosis, cardiac change, rhabdomyolysis, hepatic change, lipemia, renal change, death, previous history of epilepsy between groups. We also used MannWhitney U-test to analyze the age, numbers of AED. Analytical significance level was $p<0.05$.

\section{Results}

The propofol infusion therapy was performed in 13 patients. The method of propofol treatment was that the medial value of initial loading dose of propofol was $80 \mathrm{mg}$ (range, 0-180 mg), the median value of maximal infusion rate of propofol was $8 \mathrm{mg} / \mathrm{kg} / \mathrm{hr}$ (range, $0.3-15 \mathrm{mg} / \mathrm{kg} / \mathrm{hr}$ ), the median rate of maintenance of propofol infusion was $5.6 \mathrm{mg} / \mathrm{kg} / \mathrm{hr}$ (range, $0.3-9.7 \mathrm{mg} / \mathrm{kg} / \mathrm{hr}$ ) and median duration of propofol infusion was 78 hours (range, 8-426 hours).

Analyzing the clinical features during the status epilepticus, hypotension was in 14 (56.3\%) patients, metabolic acidosis in 7 (43.8\%), cardiac change including arrhythmia in $15(21.9 \%)$, rhabdomyolysis in $11(46.9 \%)$, hepatic change in $6(34.4 \%)$, lipid change in 8 $(18.8 \%)$, and renal change in 10 (25.0\%) (Fig. 1).

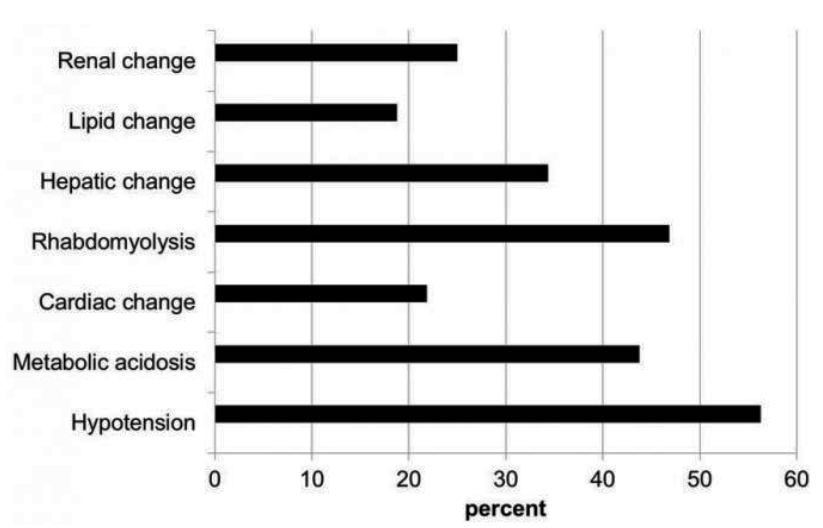

Figure 1. The change of clinical features during the status epilepticus.

When we compared the clinical features between ESE and RSE, there was no significant difference in sex, age, metabolic acidosis, cardiac change, rhabdomyolysis, hepatic change, renal change, and number of antiepileptic drugs. The death rate was higher in RSE group (38.1\%) than in ESE group (9.1\%), while the previous epilepsy history was higher in ESE group (54.5\%) than in RSE group (19.0\%). But the death rate and previous epilepsy history did not show statistical significant differences $(p \geq 0.05)$. Hypotension and dyslipidemia were much higher in RSE group $(81.0 \%, 28.6 \%)$ than in ESE group $(9.1 \%, 0.0 \%)$, which had statistical significant difference $(p<0.001, p<0.05)$ (Table 2).

According to the anesthetic treatments, we compared the clinical features of refractory status epilepticus. When we compared RSE-P group to RSE-0 group, there were not any significant differences in sex, age, hypotension, metabolic acidosis, cardiac change, rhabdomyolysis, hepatic change, lipid change, renal change, lipid change, the previous history of epilepsy and the number of antiepileptic drugs. In hypotension, RSE-P group (92.3\%) had a higher rate than RSE-0 group (62.5\%). But it did not reach statistical significant difference ( $p>0.1)$ (Table 3).

We compared the duration of admission between the groups. When we compared SE group with RSE group, the hospital days were significantly longer in RSE group (median, range; 41, 2-240) than in ESE group (median, range; 7, 3-60) ( $p=0.012)$ (Fig. 2). However, there was no significant differences of hospital days between RSE-P group (median, range; 15, 2-229) and RSE-0 group (median, range; 69, 9-240) (Fig. 3).

The outcomes after the treatment of status epilepticus showed statistically difference between ESE and RSE groups, having tendency to get worse outcome more in RSE group than in ESE group $(p=0.007)$. However they did not between RSE-P and RSE- 0 groups. 


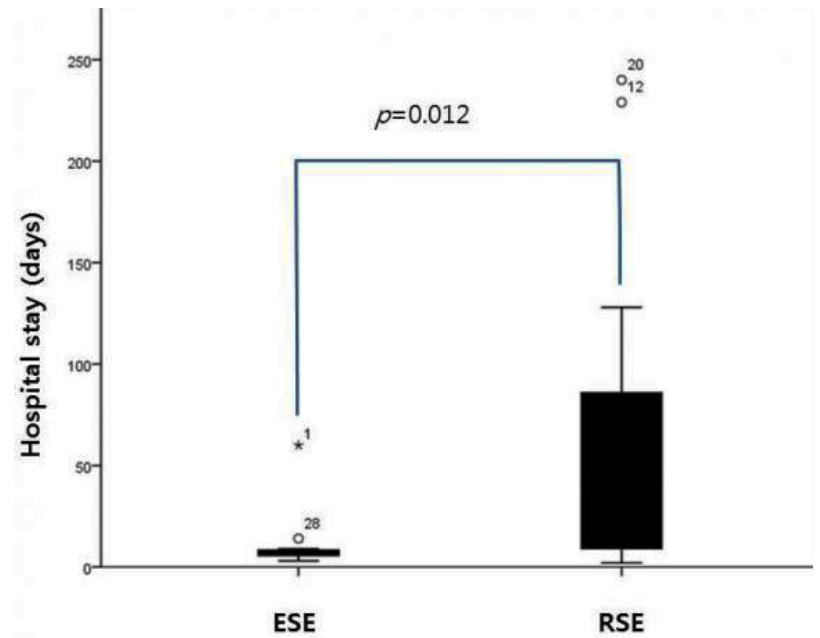

Figure 2. Comparison of hospital stays between established status epilepticus (ESE) and refractory status epilepticus (RSE). There is significant difference of hospital stay between them $(p=0.012)$.

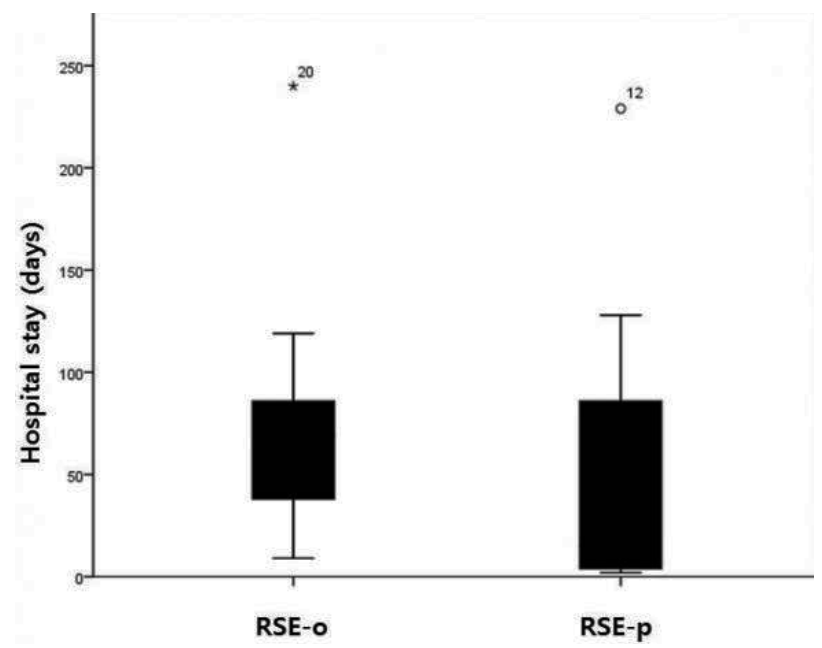

Figure 3. Comparison of hospital stays between propofol and other anesthetics groups. There is no significant difference of hospital stay between those groups ( $p>0.05)$. 'RSE-o: refractory status epilepticus, treated with the other anesthetics. RSE-p: refractory status epilepticus, treated with propofol.

According to the classification of outcome after status epilepticus treatment, RSE group had 2 (9.5\%) patients in "return to baseline", $11(52.4 \%)$ in "no return to baseline", and $8(38.1 \%)$ in "dead", while ESE group had $6(54.5 \%), 4(36.4 \%)$, and 1 (9.1\%) patients irrespectively (Fig. 4). However, when we compared RSE-P group with RSE-O group, the proportion of each category was not significant difference between them. The numbers of patients were 1 (7.7\%), 7 (53.8\%), and 13 (38.5\%) in RSE-P group irrespectively, while they were $1(12.5 \%), 4(50.0 \%)$, and, $3(37.5 \%)$ patients in

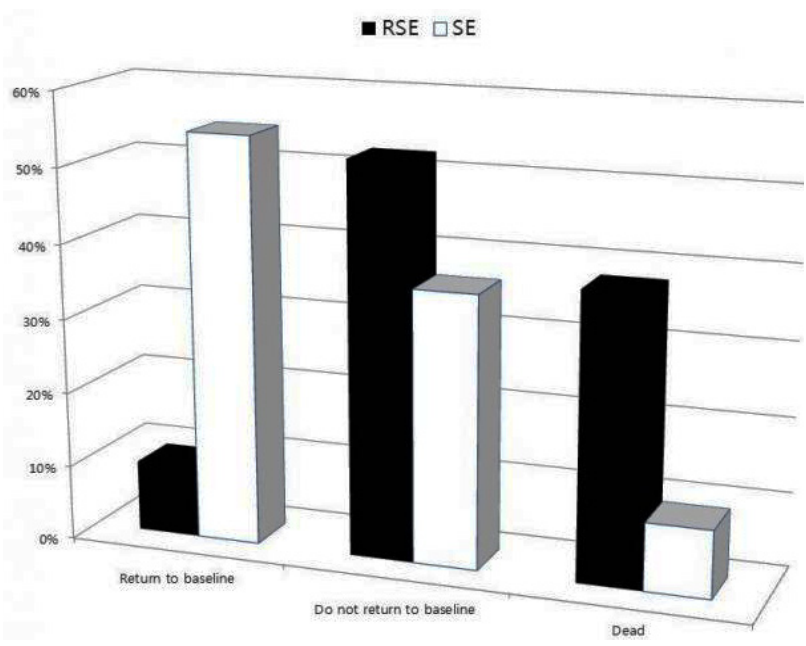

Figure 4. Comparison of outcomes after treatment between established status epilepticus (ESE) and refractory status epilepticus (RSE). There is significant difference of treatment outcome between SE and RES groups $(p=0.007)$.

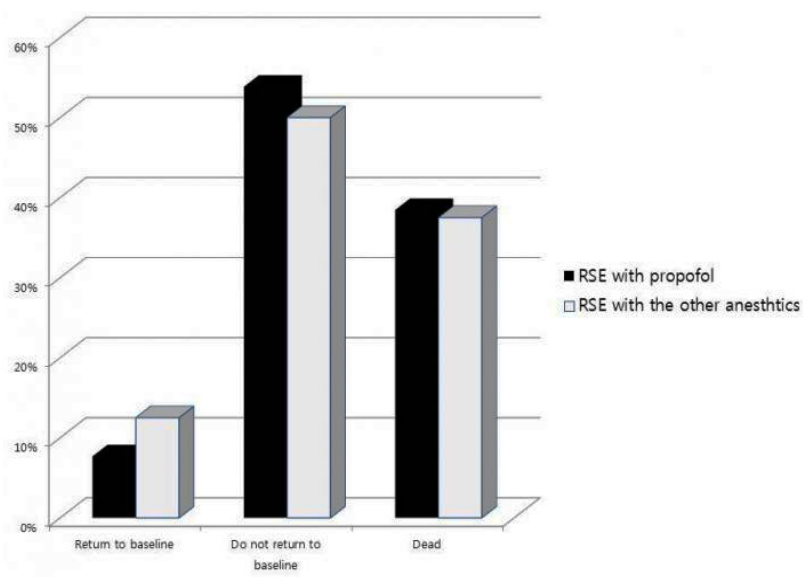

Figure 5. Comparison of outcomes after treatment between propofol and other anesthetics groups. There is no significant difference of treatment outcome between them $(p>0.1)$.

RSE-O group irrespectively (Fig. 5).

\section{Discussion}

In our study, RSE showed longer hospital days and worse treatment outcome than ESE. That is not strange results because RSE is main cause of fatality in SE. Also RSE had significantly higher rate in hypotension and dyslipidemia than ESE. Interestingly our data suggest that cardiovascular problem and lipid profile changes could be prominent clinical parameters to indicating worse prognosis in RSE. However, Comparison of clinical features between RSE-P and RSE-O 
Table 2. Comparison of clinical characteristics between status epilepticus (SE) and refractory status epilepticus (RSE) groups

\begin{tabular}{lccc}
\hline & ESE $(\mathrm{n}=11)$ & RSE $(\mathrm{n}=21)$ & $p$-value \\
\hline Male & $9(47.4)$ & $10(52.6)$ & 0.066 \\
Age [yr, median (range)] & $35(16-80)$ & $27(16-81)$ & 0.611 \\
Hypotension & $1(9.1)$ & $17(81.0)$ & 0.000 \\
Acidosis & $5(45.5)$ & $9(42.9)$ & 0.298 \\
Cardiac change & $2(18.2)$ & $5(23.8)$ & 0.544 \\
Rhabdomyolysis & $5(45.5)$ & $10(47.6)$ & 0.500 \\
Hepatic change & $2(18.2)$ & $9(42.9)$ & 0.158 \\
Lipid change & $0(0.0)$ & $6(28.6)$ & 0.017 \\
Death & $1(9.1)$ & $8(38.1)$ & 0.090 \\
Arrhythmia & $1(9.1)$ & $6(28.6)$ & 0.212 \\
Renal change & $3(30.0)$ & $5(23.8)$ & 0.575 \\
Previous epilepsy & $6(54.5)$ & $4(19.0)$ & 0.050 \\
Number of AED, median (range) & $4(1-7)$ & $5(1-8)$ & 0.628 \\
\hline
\end{tabular}

${ }^{*} \mathrm{AED}$; antiepileptic drug, numbers in the parentheses are percentile or range.

Table 3. Comparison of clinical features between propofol (RES-P) and other anesthetics (RES-O) groups

\begin{tabular}{lccc}
\hline & RSE-P $(n=13)$ & RSE-O $(n=8)$ & $p$-value \\
\hline Male & $6(46.2)$ & $4(50.0)$ & 0.608 \\
Age [yr, median (range)] & $28(17-81)$ & $25(16-61)$ & 0.221 \\
Hypotension & $12(92.3)$ & $5(62.5)$ & 0.133 \\
Acidosis & $4(30.8)$ & $5(62.5)$ & 0.166 \\
Cardiac change & $4(30.8)$ & $1(12.5)$ & 0.344 \\
Rhabdomyolysis & $6(46.2)$ & $4(50.0)$ & 0.605 \\
Hepatic change & $6(46.2)$ & $3(37.5)$ & 0.538 \\
Lipid change & $3(23.1)$ & $3(37.5)$ & 0.738 \\
Death & $5(38.5)$ & $3(37.5)$ & 0.664 \\
Arrhythmia & $5(38.5)$ & $1(12.5)$ & 0.221 \\
Renal change & $3(23.1)$ & $2(25.0)$ & 0.656 \\
Previous epilepsy & $2(15.4)$ & $2(25.0)$ & 0.498 \\
Number of AED, median (range) & $4(2-8)$ & $5(1-8)$ & 0.853 \\
\hline
\end{tabular}

*AED; antiepileptic drug, numbers in the parentheses are percentile or range.

subgroups did not show any significant differences. This means that using propofol can cause propofol infusion syndrome, but RSE itself can cause the similar clinical features to propofol infusion syndrome.

Propofol has been used since the early 1980s for anesthesia in the operation room and sedation in the intensive care unit as well as currently for refractory status epilepticus. ${ }^{18}$ It is highly lipid soluble and metabolized in the liver, and has very short distribution and short elimination half-life (30-60 min) and little propensity to accumulate. ${ }^{19}$ The mechanism of action is postulated to be the modulation of GABA receptors at a site different from that targeted by benzodiazepines and barbiturates. ${ }^{20,21}$ High dose and prolonged treatment of propofol can cause propofol infusion syndrome. Some fatal cases have been reported. ${ }^{22,23}$ Typical clinical features of propofol infusion syndrome are lactic acidosis, arrhythmia, hypotension, cardiovascular failure, liver enlargement, increased liver enzymes, rhabdomyolysis, elevated serum creatine kinase, urea and potassium, hypertriglyceridemia, renal failure, oliguria, ketonuria, green or red-colored urine. ${ }^{24}$ Among them, lipemia, metabolic acidosis, and hepatic steatosis may have been caused by lipid infusion. The byproduct of liver conversion of fatty acids to ketone bodies could potentially contribute to metabolic acidosis. Metabolic acidosis could have also been caused by hypoperfusion secondary to propofolinduced bradycardia or precipitated by other concomitant medications (e.g., vasopressors). Muscle damage is indicated by the 
increase of serum creatine kinase and direct cardiac involvement is evidenced by the increase of serum cardiac troponin I. ${ }^{14}$ According to other study, propofol infusion syndrome was defined as suddenly developed and poorly controlled cardiac arrhythmia such as sudden

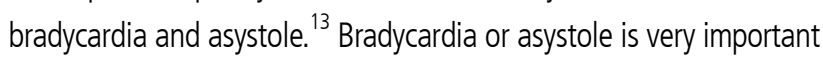
in diagnosis of propofol infusion syndrome, but it may not be unique results of using propofol. Those clinical features could be found in patients with RSE itself. So the abnormal chemistry profiles and cardiovascular changes might be considered not only in the findings of propofol treatment but also in the overall state of refractory status epilepticus.

Known risk factors of propofol infusion syndrome are using high dose propofol ( $>83 \mu \mathrm{g} / \mathrm{kg} / \mathrm{min})$, more than 48 hours, concomitant use of vasopressor. ${ }^{11,13}$ In this study, the mean infusion rate of propofol was $81.5 \mu \mathrm{g} / \mathrm{kg} / \mathrm{min}$. It was higher than the recommended dose of the ILAE guideline of treatment of SE ( $\geq 5 \mathrm{mg} / \mathrm{kg} / \mathrm{hr}){ }^{5}$ The mean duration of treatment with propofol was 153 hour. It was longer than the period of duration as one of alleged risk factors of propofol infusion syndrome..$^{9,23,25,26}$ Our use of propofol is more than enough to induce the propofol infusion syndrome. In other words, our cases are also having tendency to be very refractory to treatment.

In conclusion, our study did not demonstrate any significant differences in clinical features of propofol infusion syndrome between RSE-P and RSE-O groups. It may suggest that the clinical features of propofol infusion syndrome can be the clinical features of RSE regardless of using propofol. We need to be more careful in diagnosing propofol infusion syndrome whether those findings are due to propofol or RSE per se. This study is limited retrospective study and has the small numbers of patients, so that a more comprehensive prospective study could make the propofol infusion syndrome clear. Until further data become available, caution should be recommended when using high dose and long term propofol treatment.

\section{References}

1. Bleck TP. Management approaches to prolonged seizures and status epilepticus. Epilepsia 1999;40 Suppl 1:\$59-63.

2. Brown LA, Levin GM. Role of propofol in refractory status epilepticus. Ann Pharmacother 1998:32:1053-59.

3. Mayer SA, Claassen J, Lokin J, Mendelsohn F, Dennis U, Fitzsimmons BF. Refractory status epilepticus: frequency, risk factors, and impact on outcome. Archives of Neurology 2002;59:205-10.

4. Chen JW, Wasterlain CG. Status epilepticus: pathophysiology and management in adults. Lancet Neurology 2006;5:246-56.
5. Niermeijer JM, Uiterwaal CS, Van Donselaar CA. Propofol in status epilepticus: little evidence, many dangers? Journal of Neurology 2003; 250:1237-40.

6. Claassen J, Hirsch L, Emerson RG, Mayer SA. Treatment of refractory status epilepticus with pentobarbital, propofol, or midazolam: a systematic review. Epilepsia 2002;43:146-53.

7. Riviello JJ Jr., Claassen J, LaRoche SM, et al. Treatment of status epilepticus: an international survey of experts. Neurocrit Care 2013;18: 193-200.

8. Parviainen I, Kalviainen R, Ruokonen E. Propofol and barbiturates for the anesthesia of refractory convulsive status epilepticus: pros and cons. Neurological Research 2007;29:667-71.

9. Vasile B, Rasulo F, Candiani A, Latronico N. The pathophysiology of propofol infusion syndrome: a simple name for a complex syndrome. Intensive Care Med 2003;29:1417-25.

10. Hanna JP, Ramundo ML. Rhabdomyolysis and hypoxia associated with prolonged propofol infusion in children. Neurology 1998;50:301-3.

11. Iyer VN, Hoel $R$, Rabinstein AA. Propofol infusion syndrome in patients with refractory status epilepticus: an 11-year clinical experience. Crit Care Med 2009;37:3024-30.

12. Fudickar A, Bein B. Propofol infusion syndrome: update of clinical manifestation and pathophysiology. Minerva Anestesio/ 2009;75:339-44.

13. Fudickar A, Bein B, Tonner PH. Propofol infusion syndrome in anaesthesia and intensive care medicine. Curr Opin Anaesthesiol 2006;19: 404-10.

14. Kang TM. Propofol infusion syndrome in critically ill patients. Ann Pharmacother 2002;36:1453-56.

15. Bray RJ. Propofol-infusion syndrome in children. Lancet 1999;353:2074-5.

16. Lothman E. The biochemical basis and pathophysiology of status epilepticus. Neurology 1990;40:13-23.

17. Collins RC, Lothman EW, Olney JW. Status epilepticus in the limbic system: biochemical and pathological changes. Advances in Neurology 1983;34:277-88.

18. Rogers KM, Dwar KM, McCubbin TD, Spence AA. Preliminary experience with $\mathrm{ICl} 35868$ as an i.v. induction agent: comparison with althesin. Br J Anaesth 1980;52:807-10.

19. Stecker MM, Kramer TH, Raps EC, O'Meeghan R, Dulaney E, Skaar DJ. Treatment of refractory status epilepticus with propofol: clinical and pharmacokinetic findings. Epilepsia 1998;39:18-26.

20. Holtkamp M, Tong $X$, Walker MC. Propofol in subanesthetic doses terminates status epilepticus in a rodent model. Ann Neurol 2001;49: 260-63.

21. Peduto VA, Concas A, Santoro G, Biggio G, Gessa GL. Biochemical and electrophysiologic evidence that propofol enhances GABAergic transmission in the rat brain. Anesthesiology 1991;75:1000-9.

22. Perrier ND, Baerga-Varela Y, Murray MJ. Death related to propofol use in an adult patient. Crit Care Med 2000;28:3071-4.

23. Cremer OL, Moons KG, Bouman EA, Kruijswijk JE, de Smet AM, Kalkman CJ. Long-term propofol infusion and cardiac failure in adult 
head-injured patients. Lancet 2001;357:117-8.

24. Motsch J, Roggenbach J. Propofol infusion syndrome. Anaesthesist 2004;53:1009-22;

25. Fodale $\mathrm{V}$, La Monaca E. Propofol infusion syndrome: an overview of a perplexing disease. Drug Saf 2008;31:293-303.

26. Ahlen K, Buckley CJ, Goodale DB, Pulsford AH. The 'propofol infusion syndrome': the facts, their interpretation and implications for patient care. Eur J Anaesthesiol 2006;23:990-8. 